Proc. Indian Acad. Sci. (Earth Planet. Sci.), Vol. 94, No. 1, March 1985, pp. 71-76.

(C) Printed in India.

\title{
Analysis of gravity gradients over a thin infinite sheet
}

\author{
G D J SIVAKUMAR SINHA and H V RAM BABU \\ National Geophysical Research Institute, Hyderabad 500007, India \\ MS received 9 September 1983; revised 22 January 1985
}

\begin{abstract}
Expressions are derived for the horizontal and vertical gradients of the gravity anomaly over a thin dipping sheet of infinite depth extent. The resultant of these two gradients, known as the complex gradient, is also derived and properties of the amplitude and phase responses of the complex gradient are studied. It is shown that the amplitude plot is a symmetric curve whose shape is independent of the dip $(\theta)$ of the sheet whereas the phase plot is an antisymmetric curve with an offset value equal to $\theta-\pi / 2$. The depth to top of the sheet is obtained from the amplitude plot. The method is applied on a field example and the results are in good agreement with the results obtained by earlier workers.
\end{abstract}

Keywords. Gravity interpretation; thin sheet; complex gradient.

\section{Introduction}

In recent years, gravity gradients are being precisely measured and it was reported that the gradients have higher resolving power than the gravity itself (Hammer 1979). Recently, the term complex gradient was introduced (Atchuta Rao et al 1981, 1983; Atchuta Rao and Ram Babu 1981) which is defined as the resultant of both the horizontal and vertical gradients of gravity or magnetic anomaly. The present paper describes a method to evaluate the parameters of a thin sheet of infinite depth extent by analysing the amplitude and phase plots of the complex gradient of its gravity anomaly.

\section{Theory}

Let there be a thin sheet (figure 1) of infinite depth extent whose upper edge is buried at a depth $h$. If $\theta$ is the dip and $\sigma$ is the density contrast of the sheet to its surroundings, the expression for the horizontal and the vertical gradients of the gravity anomaly over the thin sheet may be derived from the expression for the gravity anomaly over a thin sheet of finite depth extent as follows.

The expression $(\Delta g(x))$ for the gravity anomaly over a thin sheet of finite depth extent is given by Rao and Murthy (1978) as follows:

$$
\begin{aligned}
& \Delta g(x)=2 G \sigma t\left[\frac{1}{2} \sin \theta \ln \frac{(x-a)^{2}+H^{2}}{x^{2}+h^{2}}\right. \\
& \left.+\cos \theta\left(\tan ^{-1} \frac{x}{h}-\tan ^{-1} \frac{x-a}{H}\right)\right],
\end{aligned}
$$

in which $G$ is the universal gravitational constant, $\sigma$ is the density contrast, $t$ is the thickness of the sheet, $\theta$ is the dip of the sheet, $h$ is the depth to the upper edge of the 
sheet, $H$ is the depth to the lower edge of the sheet, and $a=(H-h) / \tan \theta$.

The horizontal $\left(\Delta g_{x}(x)\right)$ and the vertical $\left(\Delta g_{z}(x)\right)$ gradients of the gravity anomaly over a thin sheet of finite depth extent may be written from equation (1) as follows:

$$
\Delta g_{x}(x)=M\left[\frac{h \cos \theta-x \sin \theta}{x^{2}+h^{2}}+\frac{(x-a) \sin \theta-H \cos \theta}{(x-a)^{2}+H^{2}}\right],
$$

and

$$
\Delta g_{z}(x)=M\left[\frac{H \sin \theta+(x-a) \cos \theta}{(x-a)^{2}+H^{2}}-\frac{x \cos \theta+h \sin \theta}{x^{2}+h^{2}}\right],
$$

where $M=2 G \sigma t$. When the sheet extends to infinity in the down dip direction, i.e., $H \rightarrow \infty$, equations (2) and (3) may be re-written as follows:

$$
\Delta g_{x}(x)=M \frac{h \cos \theta-x \sin \theta}{x^{2}+h^{2}}
$$

and

$$
\Delta g_{z}(x)=-M \frac{x \cos \theta+h \sin \theta}{x^{2}+h^{2}} .
$$

Equations (4) and (5) are the expressions for the horizontal and the vertical gradients, respectively, of the gravity anomaly over a thin sheet of infinite depth extent. The horizontal and vertical gradients of the gravity anomaly over an assumed sheet with $h$ $=10 \mathrm{~m} ; \theta=60^{\circ}$ and $P=100$ c.g.s. units is shown in figure 1 .

The complex gradient $\overline{\Delta g_{c}}$ of $\Delta g$ is defined by Atchuta Rao et al (1981) as follows:

$$
\overline{\Delta g_{c}}(x)=\Delta g_{z}(x)+j \Delta \dot{g}_{x}(x) \text {. }
$$

The amplitude $A(x)$ and phase $\phi(x)$ of $\overline{\Delta g_{c}}(x)$ are given by

and

$$
A(x)=\left[\Delta g_{x}^{2}(x)+\Delta g_{z}^{2}(x)\right]^{1 / 2},
$$

$$
\Phi(x)=\tan ^{-1}\left[\Delta g_{x}(x) / \Delta g_{z}(x)\right]
$$

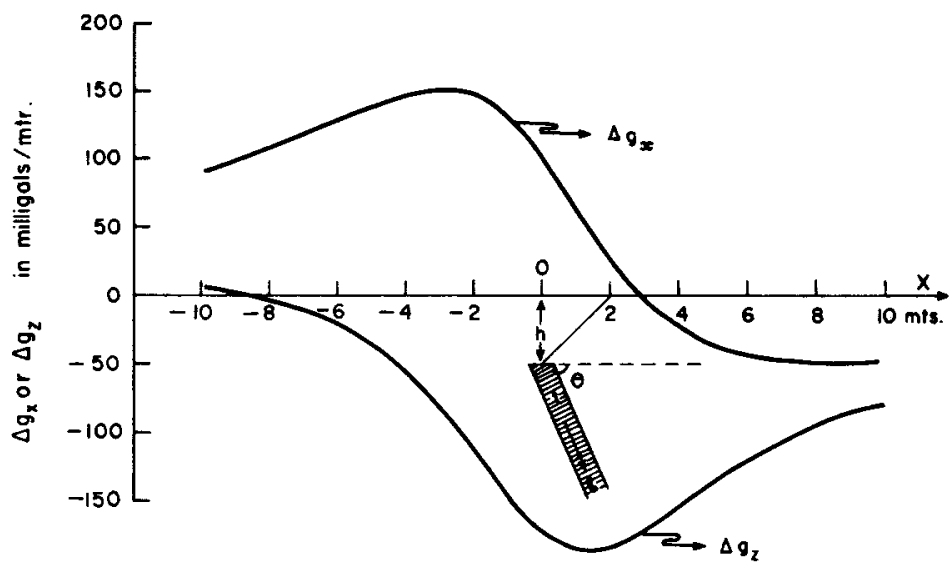

Figure 1. The horizontal $\left(\Delta g_{x}(x)\right)$ and the vertical $\left(\Delta g_{z}(x)\right)$ gradients of the gravity anomaly over a thin sheet of infinite depth extent. 
From equations (3) through (6) we write the complex gradient for the gravity anomaly due to the thin infinite sheet as

$$
\overline{\Delta g_{c}}(x)=\frac{-M}{x^{2}+h^{2}}[(x \cos \theta+h \sin \theta)-j(h \cos \theta-x \sin \theta)] \text {. }
$$

From equations (4), (5), (7) and (8), the amplitude $A(x)$ and phase, $\Phi(x)$ of $\overline{\Delta g_{c}}(x)$ may be written as follows:

and

$$
A(x)=M /\left(x^{2}+h^{2}\right)^{1 / 2},
$$

$$
\Phi(x)=\tan ^{-1}\left[\frac{x \sin \theta-h \cos \theta}{x \cos \theta+h \sin \theta}\right]
$$

The theoretical amplitude $A(x)$ and phase $\Phi(x)$ of the complex gradient of the gravity anomaly over an infinite sheet with $h=10 \mathrm{mtrs}, \theta=60^{\circ}$ and $P=100$ c.g.s. units is shown in figure 2.

\subsection{Analysis of $A(x)$ and $\Phi(x)$}

It may be noted from (10) that the amplitude function $A(x)$ is even and hence a plot of $A(x)$ versus $x$ will be a symmetric curve with respect to the origin, $x=0$. $A(x)$ attains its maximum value directly over the origin. The maximum value of $A(x)$ is given by the following equation.

$$
\left.A(x)\right|_{x=0}=M / h=A_{\max } .
$$

At $x= \pm h=X_{1}$, the amplitude is as follows:

$$
\left.A(x)\right|_{x= \pm h}=\frac{M}{h \sqrt{2}}=0.7072 A_{\max }
$$

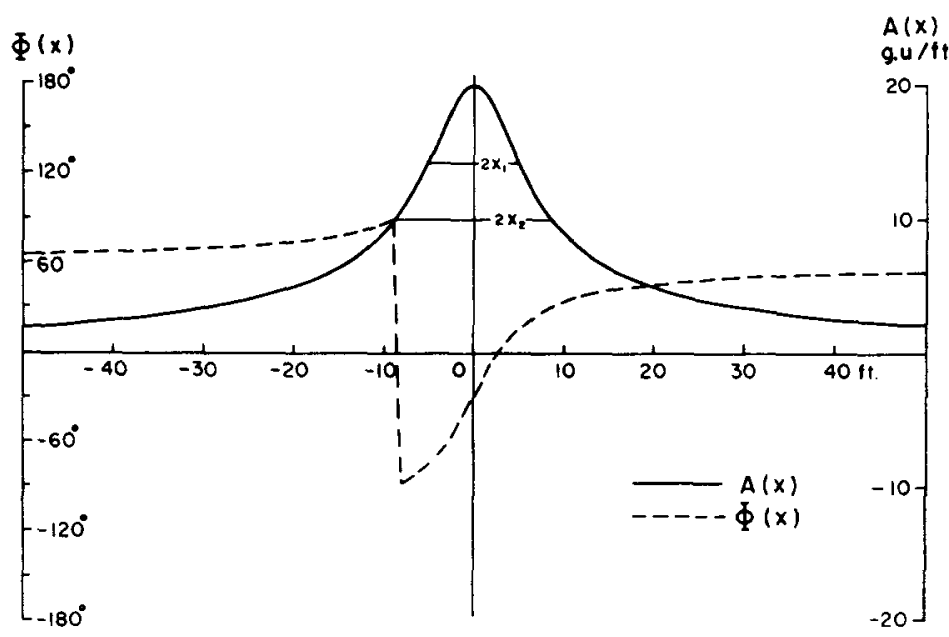

Figure 2. Amplitude $[A(x)]$ and phase $[\Phi(x)]$ plots of complex gradient of the gravity anomaly over $a$ thin sheet. 
It is also easy to derive from (10) that $A(x)$ falls off to half its maximum value when $x$ $= \pm h \sqrt{ } 3=X_{2}$

i.e. $\left.\quad A(x)\right|_{x= \pm h \sqrt{ } 3}=\frac{M}{2}=0.5 A_{\max }$.

The following properties of the phase function $\Phi(x)$ may be derived from (11).

$$
\left.\Phi(x)\right|_{x=0}=\theta-\pi / 2=\Phi_{0} \text {. }
$$

and

$$
\Phi(x)+\Phi(-x)=2 \theta \text {. }
$$

Using the above properties of $A(x)$ and $\Phi(x)$ the parameters of the sheet may be evaluated in the following way.

\section{Practical approach}

Let there be a gravity anomaly $(\Delta g)$ assumed to be due to a sheet-like structure extending to a great depth. The horizontal gradient $\Delta g_{x}$ of $\Delta g(x)$ may be calculated using the following formula:

$$
\Delta g_{x}(x)=[\Delta g(x+\Delta x)-\Delta g(x-\Delta x)] /(2 \Delta x),
$$

where $\Delta x$ is the interval of observations.

If the vertical gradient measurements are not available, they may be computed using the Hilbert transform as the vertical gradient of the gravity anomaly over a twodimensional structure is the Hilbert transform of the horizontal gradient (Stanley and Green 1976). The amplitude and phase functions of the complex gradient are computed using (7) and (8) and are plotted on linear scales. The amplitude plot will be a symmetric curve whose maximum occurs directly over the origin. On the amplitude plot, the distances $X_{1}$ and $X_{2}$ corresponding to $70 \%$ and $50 \%$ maximum amplitude of $A(x)$ are read and using the properties given by (13) and (14) the depth $(h)$ to the top may be found. The value of the phase function directly over the origin is given by (15) from which the $\operatorname{dip}(\theta)$ of the sheet may be calculated. The amplitude factor $M$ may be evaluated using the property given by (12) from which the thickness $(t)$ of the sheet may be evaluated by assuming the density contrast.

\section{Field example}

The gravity anomaly across the Moburn ore body (Noranda, Quebec) analysed by Grant and West (1965, figure 10-10), using the Ribbon Model is shown in figure 3. The horizontal and vertical gradients of this anomaly are computed which are also shown in figure 3. The amplitude and phase plots (figure 4) of the complex gradient are found and analysed to yield the parameters given in table 1 . The thickness of the sheet is evaluated using a density contrast of $1.9 \mathrm{~g} / \mathrm{cm}^{3}$. The depth to top of the ore body obtained by Roy (1966) using the method of continuation is also tabulated for comparison. A comparison of results given in table 1 indicates that the present method provides reasonably accurate results of interpretation. 


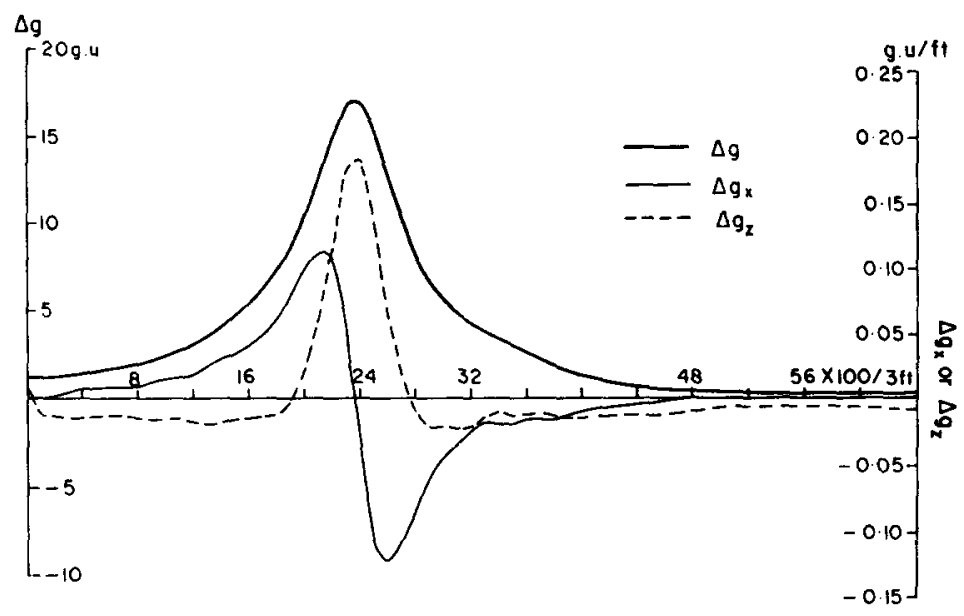

Figure 3. Gravity anomaly $(\Delta g)$ over Moburn ore body and its horizontal $\left(\Delta g_{x}\right)$ and vertical $\left(\Delta_{g_{z}}\right)$ gradients.

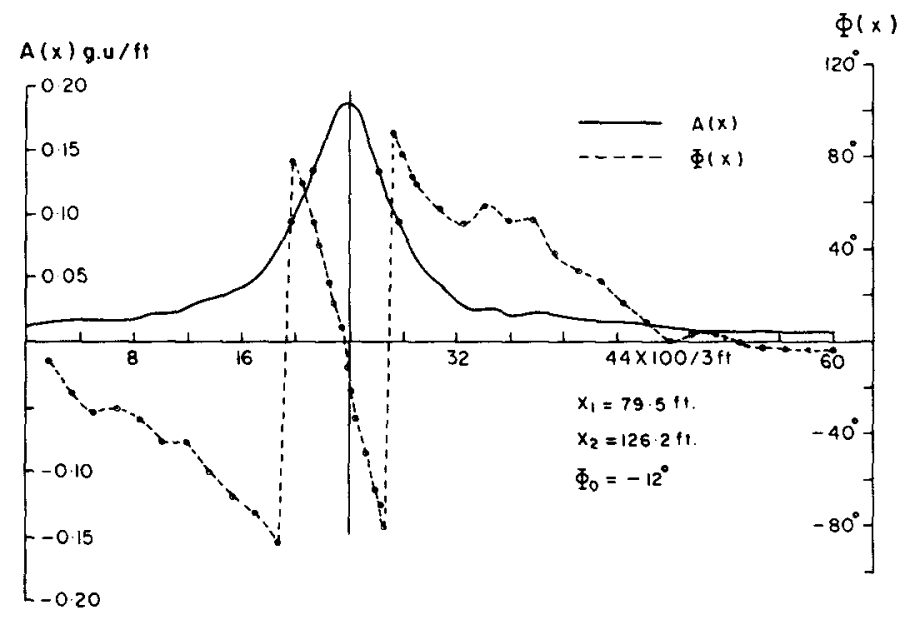

Figure 4. Amplitude $A(x)$ and phase $\Phi(x)$ plots of the complex gradient of the gravity anomaly shown in figure 3 .

Table 1. Comparison of results obtained by using the present method with those obtained by other methods.

\begin{tabular}{lllll}
\hline Parameter & $\begin{array}{l}\text { Present } \\
\text { method }\end{array}$ & $\begin{array}{l}\text { Grant and } \\
\text { West (1965) }\end{array}$ & $\begin{array}{l}\text { Roy } \\
(1966)\end{array}$ & Drilling \\
\hline Depth to top & $80 \mathrm{ft}$ & $56 \mathrm{ft}$ & $100 \mathrm{ft}$ & $100 \mathrm{ft}$ \\
Thickness & $\begin{array}{l}79 \mathrm{ft} \\
116 \mathrm{ft}\end{array}$ & - & - \\
Dip $(\theta)$ & $79^{\circ} \mathrm{N}$ & $83^{\circ} \mathrm{N}$ & - & - \\
\hline
\end{tabular}




\section{Acknowledgements}

The authors thank Dr D Atchuta Rao for encouragement.

\section{References}

Atchuta Rao and Ram Babu H V 1981 Geoviews 941

Atchuta Rao D, Rambabu H V and Sanker Narayan P V 1981 Geophysics 461572

Atchuta Rao D, Sivakumar Sinha G D J and Ram Babu H V 1983 Journal of Association of Exploration Geophysicists 425

Grant F S and West G F 1965 Interpretation theory in applied geophysics (New York: McGraw Hill) Hammer S 1979 Geophysics 4499

Rao B S R and Murthy I V R 1978 Gravity and magnetic methods of prospecting (New Delhi: Arnold Heinemann)

Roy A 1966 Geoexploration 465

Stanley J M and Green R 1976 Geophysics 411370 